\title{
Binge eating disorder: a symptom-level investigation of genetic and environmental influences on liability
}

\author{
K. S. Mitchell 1,2 , \\ Mazzeo ${ }^{1,2,6,{ }^{*}}$ \\ M. C. Neale $2,3,4$, \\ C. M. Bulik 5 , \\ S. H. Aggen 2,3, \\ K. S. Kendler 2,3 , and S. E. \\ ${ }^{1}$ Department of Psychology, Virginia Commonwealth University, Richmond, VA, USA \\ 2 Virginia Institute for Psychiatric \& Behavioral Genetics, Virginia Commonwealth University, \\ Richmond, VA, USA \\ ${ }^{3}$ Department of Psychiatry, Virginia Commonwealth University, Richmond, VA, USA \\ ${ }^{4}$ Department of Human Genetics, Virginia Commonwealth University, Richmond, VA, USA \\ ${ }^{5}$ Departments of Psychiatry and Nutrition, University of North Carolina, Chapel Hill, NC, USA \\ ${ }^{6}$ Department of Pediatrics, Virginia Commonwealth University, Richmond, VA, USA
}

\begin{abstract}
Background-Recent behavioral genetic studies have emphasized the importance of investigating eating disorders at the level of individual symptoms, rather than as overall diagnoses. We examined the heritability of binge eating disorder (BED) using an item-factor analytic approach, which estimates contributions of additive genetic (A), common environmental (C), and unique environmental (E) influences on liability to BED as well as individual symptoms.

Method-Participants were 614 monozygotic and 410 dizygotic same-sex female twins from the Mid-Atlantic Twin Registry who completed a self-report measure of BED symptoms based upon DSM-IV criteria. Genetic and environmental contributions to BED liability were assessed at the diagnostic and symptom levels, using an item-factor approach.
\end{abstract}

Results-Liability to BED was moderately heritable; $45 \%$ of the variance was due to A, with smaller proportions due to C (13\%), and E (42\%). Additive genetic effects accounted for 29-43\% of the variance in individual items, while only $8-14 \%$ was due to $\mathrm{C}$.

Conclusions-Results highlight the relevance of examining eating disorders at the symptom level, rather than focusing on aggregate diagnoses.

\section{Keywords}

Binge eating disorder; heritability; item-factor model; twins

\section{Introduction}

Eating disorders are complex and multi-determined illnesses, which are influenced by genetic as well as environmental factors (Striegel-Moore \& Bulik, 2007). Twin studies, which partition

\footnotetext{
*Address for correspondence to: Dr S. E. Mazzeo, Department of Psychology, Virginia Commonwealth University, PO Box 842018, Richmond, VA 23284-2018., semazzeo@vcu.edu.

Declaration of Interest

None.
} 
the variance of a given trait or disorder into additive genetic (A), common environmental (i.e. factors shared among members of a twin pair, or C), and unique environmental (i.e. factors not shared by co-twins, or E) influences, have provided significant insight into the influence of genes and environment on anorexia nervosa (AN) and bulimia nervosa (BN; see Bulik et al. 2000, for a review). However, only one published study (Javaras et al. 2008) has investigated the heritability of binge eating disorder (BED) specifically. These authors found that, in two separate samples (one from the USA, and one from Norway), additive genetic factors significantly contributed to BED liability. In both samples, the best-fitting model included only additive genetic and unique environmental components; common environment was not significantly associated with BED liability. Variance estimates in the US sample were $\mathrm{a}^{2}=0.57$ (95\% CI 0.30-0.77) and $\mathrm{e}^{2}=0.43$. In the Norwegian sample, results were comparable $\left(\mathrm{a}^{2}=0.39\right.$ and $\left.\mathrm{e}^{2}=0.61\right)$.

Although the Javaras et al. (2008) study was the first to investigate the genetic epidemiology of the DSM-IV diagnosis of BED (APA, 2000), a small number of prior investigations evaluated the heritability of specific aspects of the disorder. Reichborn-Kjennerud et al. (2003) investigated binge eating with loss of control and found that among females, $48 \%$ of the variance in binge eating was accounted for by additive genetic factors (95\% CI 0.17-0.60); $3 \%$ was associated with common environment (95\% CI $0.00-0.29)$, and $49 \%$ was associated with unique environmental factors (95\% CI 0.40-0.59). In a subsequent study, Reichborn-

Kjennerud and colleagues (2004) examined binge eating occurring with loss of control and in the absence of compensatory behaviors. They found that $41 \%$ of the variance in this phenotype, which more closely approximated BED, was due to additive genetic influences (95\% CI 0.310.50 ), and $59 \%$ was associated with unshared environment (95\% CI 0.50-0.69).

The current study attempts to extend this previous work on the genetic epidemiology of BED first by replicating Javaras and colleagues' (2008) study and evaluating A, C, and E influences on the latent liability to BED, as assessed by DSM-IV criteria (APA, 2000). A second aim of the current study is to investigate genetic and environmental influences on the specific symptoms of BED, which include binge eating, loss of control, frequency and duration of binges, binge characteristics (e.g. eating until feeling uncomfortably full), distress regarding binge eating, and the absence of compensatory behaviors. Previous item-level studies of AN (Mazzeo et al. 2009) and BN (Mazzeo et al. in press) found that heritability point estimates for individual symptoms of these disorders varied in magnitude.

Item-level biometric analyses can provide valuable information about the overall disorder. For example, if the criteria for a specific diagnosis vary in their respective genetic and environmental contributions to liability, use of a sum score can provide misleading results about the overall heritability of a given diagnosis. Use of sum scores can result in a loss of information, as a composite score is rarely a 'pure' indicator of a latent trait (Neale et al. 2005; Van den Berg et al. 2007). Further, data regarding environmental and genetic influences on specific eating-disorder symptoms can be quite useful regarding assessment and diagnosis of such complex phenotypes.

Symptom-level analysis might be particularly important in the assessment of BED, as the criteria for this diagnosis remain debated. For example, as Bulik and colleagues (2007) and others (Latner \& Clyne, 2008; Striegel-Moore \& Franko, 2008) have noted, terms such as 'binge' and 'loss of control' are difficult for clinicians, patients, and researchers to operationalize. Further, as is the case for BN, few empirical data are available to support the validity of the current binge frequency and duration standards (Bulik et al. 2007; Latner \& Clyne, 2008; Striegel-Moore \& Franko, 2008). Thus, the current study uses an item-factor model to investigate BED (see Fig. 1). This model estimates overall A, C, and E influences on 
the latent continuous BED construct as well as A, C, and E contributions to each item assessing BED.

\section{Method}

\section{Participants}

Twins in this study are from the population-based Virginia Twin Registry (VTR; Kendler \& Prescott, 1999), which now constitutes part of the Mid-Atlantic Twin Registry (MATR). A complete description of this sample is provided elsewhere (Mazzeo et al. 2006, in press). The current study focuses on monozygotic (MZ, $n=614$ ) and (dizygotic, DZ, $n=410)$ same-sex female twins. Participants' mean age was 40.44 years (S.D.=8.34).

\section{Measures}

BED symptoms-Items assessing BED, based on DSM-IV criteria (APA, 2000) and adapted from the Structured Clinical Interview for DSM-IV (SCID-I; First et al. 1997), were administered in a self-report questionnaire (see Table 1 for an item summary). If participants reported no lifetime experiences of binge eating, they skipped all subsequent items related to this behavior. A total of 207 women endorsed binge-eating behavior. This item was scored dichotomously; all other items were assessed using ordinal scales. The item evaluating binge frequency per month used a free-response format. These responses were trichotomized $(0-1$, $2-7$, and $\geq 8$ binges per month) for model-fitting purposes. Seven binary items assessing compensatory behaviors following a binge were summed to create one item, in order to assess the criterion of absence of these behaviors.

\section{Statistical analyses}

Biometric modeling, which utilizes twin data to decompose the variance of a given trait or traits into additive genetic (A), common environmental (C), and unique environmental (E) influences, was conducted using Mx (Neale et al. 2003). Additive genetic effects are assumed to contribute twice as much to the covariance between MZ twins as DZ twins because, generally, MZ twins share all of their genes, while DZ twins share approximately half. Of note, this assumes an absence of genetic dominance, epistasis, or assortative mating, all of which can influence heritability estimates obtained from twin models (Plomin et al. 2008). For example, assortative mating may lead to an overestimation of the influence due to $\mathrm{C}$, and to an underestimation of variance due to A, in the classic twin model. Further, the presence of epistasis may lead to either an over- or under-estimation of A (see Keller et al. 2008, for a review). Shared environmental influences are assumed to be equivalent for $\mathrm{MZ}$ and $\mathrm{DZ}$ twins, while specific environmental influences are assumed to be uncorrelated for both MZ and DZ twin pairs.

An item-factor analytic modeling approach (Neale et al. 2006a) was used to investigate BED symptoms. This method, as described in detail elsewhere, has been applied to the analysis of AN (Mazzeo et al. 2009) and BN symptoms (Mazzeo et al. in press). In this model, each item's observed variance is decomposed into two types: that shared with the latent factor and a residual component (item-specific variance plus measurement error). Residual variance for each item was partitioned into $\mathrm{A}, \mathrm{C}$, and $\mathrm{E}$ influences. Confidence intervals (CIs) were estimated to examine the statistical precision of the $\mathrm{A}, \mathrm{C}$, and $\mathrm{E}$ parameters.

Due to the design of the survey, (which involved skip patterns related to binge eating, as noted above), not all participants provided data for each item. Selection effects resulting from the use of 'stem' items (in this case, binge eating) can impact covariance estimates, which are essential for fitting the factor model (Neale et al. 2006a). However, maximum-likelihood joint analysis of data from stem and follow-up questions from twin pairs overcomes this problem, 
because the covariance between the stem item and the co-twin's follow-up items is available (Neale et al. 2006b).

Total heritability for each individual item (i) was computed as the product of the item's squared factor loading $(\lambda)$ and $\mathrm{a}^{2}$ for the latent trait, added to the product of one minus the item's squared factor loading and the amount of the item's residual variance due to A. This equation, where $\lambda_{\mathrm{i}}$ is the factor loading for the $i$ th item is as follows:

$$
\left(\lambda_{i}^{2}\right)\left(\mathrm{a}^{2}\right)+\left(1-\lambda_{i}^{2}\right)\left(\mathrm{A}_{i}\right) .
$$

Similarly, total shared and unique environmental influences on each item were computed using this equation, respectively substituting $\mathrm{c}^{2}$ or $\mathrm{e}^{2}$ and residual variance due to $\mathrm{C}$ or $\mathrm{E}$.

\section{Results \\ BED}

A total of 32 women met criteria for BED. An ACE model, using MML estimation, was fitted to the BED data. The MZ correlation ( $r=0.58)$ was greater than the DZ correlation $(r=0.35)$, providing evidence for additive genetic effects. The largest proportion of variance was due to A $\left(\mathrm{a}^{2}=0.45,95 \%\right.$ CI $\left.0.00-0.92\right)$, followed by $\mathrm{E}\left(\mathrm{e}^{2}=0.42,95 \%\right.$ CI $\left.0.00-1.0\right)$. Last, C contributed little to the variance in liability to BED $\left(c^{2}=0.13,95 \%\right.$ CI $\left.0.00-0.96\right)$.

The CIs for $\mathrm{A}$ and $\mathrm{C}$ included 0 , indicating that $\mathrm{A}$ and $\mathrm{C}$ individually were non-significant. However, wide CIs can be a result of sparse data. Given the current study's sample size, parameters from the full ACE model are more likely to represent the true model than either submodel (Sullivan \& Eaves, 2002). The fit statistics for this model were as follows: Akaike's Information Criterion $=-51.68,-2 \log$-likelihood $(2225 \mathrm{df})=4398.32$.

This model also provides information about the variance of, and covariance among, BED criteria, through the estimation of factor loadings and residual variances, which are also partitioned into A, C, and E. All factor loadings were high in magnitude, ranging from 0.62 to 0.93 (see Table 2).

The majority of the variance in all items was due to E, except for item 5, which had a slightly higher proportion of variance due to A. Two items (3 and 5), assessing loss of control during binges and distress due to bingeing, had heritability estimates of 0.43 . Proportions of variance due to A ranged from $0.37-0.38$ for binge eating (item 1), frequency of binges (item 2), and binge characteristics (item 6). The remaining two items (4 and 7), assessing duration of binges and the absence of compensatory behaviors, had heritability point estimates of 0.33 and 0.29 , respectively. In addition, item 7 had the largest proportion of variance due to $C(19 \%)$, relative to the other items; however, as can be seen in Table 2, no items had a large proportion of variance accounted for by common environment.

\section{Discussion}

This study was the first to investigate genetic and environmental influences on liability to BED using an item-factor approach. Use of this methodology enables examination of this disorder in three distinct ways. First, the factor loadings provide data regarding how strongly each symptom is related to a single underlying construct (or diagnosis, in this case). Second, the biometric analysis of the liability to BED enables comparison of current results with those derived from the other extant study of BED at this level (Javaras et al. 2008). Third, the itemlevel analysis of the additive genetic, common environment and specific environment 
influences on specific BED symptoms indicates whether these individual components of BED are differentially influenced by these etiological factors.

The factor analysis results indicated that each of the DSM-IV criteria for BED was strongly related to the underlying latent liability to BED. It is particularly noteworthy that these factor loadings were higher than those previously obtained in symptom-level analyses of AN and BN (Mazzeo et al. 2009, in press). This suggests that each of these items is strongly related to a unidimensional latent construct. Thus, although specific controversies about the BED diagnosis remain, such as difficulties with operationally defining binge eating and determining appropriate cut-offs for binge frequency and duration, each of these symptoms does appear to capture a significant degree of variance in liability to this diagnosis.

Biometric analysis indicated that overall liability to BED was moderately influenced by additive genetic and unique environmental factors. Our CIs for BED variance components were quite wide. Indeed, they were much wider for the overall disorder than for the symptoms, probably because each item was more likely to be endorsed individually than altogether, reflecting the low prevalence of BED in this sample. However, the magnitude of the variance components estimates obtained in the current study are consistent with those obtained in the only other previous study of the heritability of BED (Javaras et al. 2008), and provide additional evidence of the role of genetic factors in the etiology of this disorder. Current findings are also consistent with a previous investigation of broadly defined BN (Bulik et al. 1998) which found that an AE model provided the best fit to the data. However, it should be noted that although these additive genetic effects on liability to BED were significant, their absolute value was moderate. Thus, it is important that researchers continue to investigate potential gene $\times$ environment interactions and correlations that might be particularly relevant to individuals with high genetic risk for BED.

Item-level analyses suggest that the specific/unique aspects of the BED symptoms all appeared to be best accounted for by a mixture of moderately strong additive genetic factors and unique environmental influences. The results differ somewhat from the previous item-level analyses conducted on the component symptoms of BN (Mazzeo et al. in press) and AN (Mazzeo et al. 2009), which found greater variability in estimates of A, C and E among specific diagnostic criteria. For example, in the item-factor analysis of BN, it was found that vomiting was more strongly influenced by additive genetic factors than the other BN symptoms. In contrast, the undue influence of weight on self-evaluation was more strongly influenced by common environmental factors than the other BN symptoms.

Overall, current results highlighting the role of the unique environment in liability to BED symptoms are consistent with those of Wade et al. (2006), which found that this component of variance was significantly associated with liability to the number of lifetime eatingdisordered behaviors endorsed in the Australian Twin Registry. Subsequent studies should investigate specific experiences which have been proposed as triggers for the development of eating disorders, particularly among individuals with known genetic risk for AN, BN, or BED, including trauma and encouragement to diet (Striegel-Moore \& Kearney-Cooke, 1994; Pike et al. 2006).

Importantly, however, the unique environment variance component includes measurement error. This should be considered in the interpretation of the results, and might also relate to some of the small differences identified among specific symptoms. For example, the item assessing the various compensatory behaviors (item 7) includes a range of behaviors (participants had to report not using these behaviors to meet BED criteria). It is interesting that the lowest $\mathrm{a}^{2}(0.29)$ and the second largest $\mathrm{e}^{2}(0.52)$ values were obtained for this item. The unique environment component of variance was also relatively high for items assessing binge 
characteristics (item $6, \mathrm{e}^{2}=0.56$ ), the duration of binge-eating behaviors (item $4, \mathrm{e}^{2}=0.57$ ), and their frequency (item $2, \mathrm{e}^{2}=0.52$ ). Several authors have noted that appropriate thresholds for specific BED criteria, including binge frequency and duration, are difficult to define empirically (e.g. Bulik et al. 2007; Latner \& Clyne, 2008; Striegel-Moore \& Franko, 2008). This difficulty in clearly defining criteria may increase measurement error for these items, thereby contributing to the size of the unique environmental component found for several items in the current study.

Several limitations of this study should be noted. First, because the sample included only European-American female twins, results might not generalize to men, non-twins, or members of other cultural groups. Second, like most twin studies, our sample includes a greater proportion of MZ twins, and individuals with relatively high levels of education (Lykken $e t$ al. 1987). Third, like many large-scale population-based twin studies, this investigation relied on self-report questionnaire data. Despite the limitations of this method, extant research has demonstrated acceptable levels of agreement between self-report, interview, and daily monitoring data in the assessment of eating disorders (e.g. Grilo et al. 2001). Last, single-item measures and 'stem' questions were used to evaluate BED symptoms, and symptoms were assessed over the lifespan. These measurement issues can contribute to attenuated reliability, decrease power, and increased recall bias (e.g. Crocker \& Algina, 1986), which are potential confounds. However, the survey was designed in this manner to maximize reliability and validity while also limiting participant burden in this large population-based study.

Despite these possible limitations, this study has several strengths, including the use of a large, population-based sample, and the use of methodology which enabled examination of the factor structure as well as symptom and diagnostic-level modeling. Diagnostic criteria for psychiatric diagnoses in general, and eating disorders in particular, continue to be debated. These methods are useful for informing assessment and diagnostic techniques, particularly in the case of complex phenotypes. In the current study, BED symptoms were strongly related to latent liability to the diagnosis, providing support for the prevailing conceptualization of BED as a distinct diagnosis.

\section{Acknowledgments}

This research was supported by the National Institutes of Health Grants MH-068520 (Mazzeo), MH-20030 (Mitchell, Neale PI), MH-001553 (Bulik PI), MH-65322 (Aggen \& Neale; Neale PI), and MH-40828 (Kendler). We are very grateful to the twins for their participation.

\section{References}

APA. Diagnostic and Statistical Manual of Mental Disorders. 4. American Psychiatric Association; Washington, DC: 2000. text-revision

Bulik CM, Brownley KA, Shapiro JR. Diagnosis and management of binge eating disorder. World Psychiatry 2007;6:142-148. [PubMed: 18188431]

Bulik CM, Sullivan PF, Kendler KS. Heritability of binge-eating and broadly defined bulimia nervosa. Biological Psychiatry 1998;44:1210-1218. [PubMed: 9861464]

Bulik CM, Sullivan PF, Wade TD, Kendler KS. Twin studies of eating disorders : a review. International Journal of Eating Disorders 2000;27:1-20. [PubMed: 10590444]

Crocker, L.; Algina, J. Introduction to Classical and Modern Test Theory. Wadsworth Group; Belmont, CA: 1986.

First, MB.; Spitzer, RL.; Gibbon, M.; Williams, JBW. Clinician Version. American Psychiatric Press; Washington, DC: 1997. Structured Clinical Interview for DSM-IV Axis I Disorders (SCID-I).

Grilo CM, Masheb RM, Wilson GT. A comparison of different methods for assessing the features of eating disorders in patients with binge eating disorder. Journal of Consulting and Clinical Psychology 2001;69:317-322. [PubMed: 11393608] 
Javaras KN, Laird NM, Reichborn-Kjennerud T, Bulik CM, Pope HG, Hudson JI. Familiality and heritability of binge eating disorder : results of a case-control family study and a twin study. International Journal of Eating Disorders 2008;41:174-179. [PubMed: 18095307]

Keller MC, Medland SE, Duncan LE, Hatemi PK, Neale MC, Maes HHM, Eaves LJ. Modeling extended twin family data I : description of the cascade model. Twin Research and Human Genetics 2008;12:818. [PubMed: 19210175]

Kendler K, Prescott C. A population-based twin study of lifetime major depression in men and women. Archives of General Psychiatry 1999;56:39-44. [PubMed: 9892254]

Latner JD, Clyne C. The diagnostic validity of the criteria for binge eating disorder. International Journal of Eating Disorders 2008;41:1-14. [PubMed: 17922537]

Lykken DT, McGue M, Tellegen A. Recruitment bias in twin research : the rule of two-thirds reconsidered. Behavior Genetics 1987;17:343-362. [PubMed: 3675526]

Mazzeo SE, Landt-Slof MCTO, Jones I, Mitchell KS, Kendler KS, Neale MC, Aggen SH, Bulik CM. Associations among post-partum depression, eating disorders and perfectionism in a populationbased sample of adult women. International Journal of Eating Disorders 2006;39:202-211. [PubMed: 16498586]

Mazzeo SE, Mitchell KS, Bulik CM, Aggen S, Kendler KS, Neale MC. A twin study of specific bulimia nervosa symptoms. Psychological Medicine. (in press).

Mazzeo SE, Mitchell KS, Bulik CM, Reichborn-Kjennerud T, Kendler KS, Neale MC. Assessing the heritability of anorexia nervosa symptoms using a marginal maximal likelihood approach. Psychological Medicine 2009;39:463-473. [PubMed: 18485259]

Neale, MC.; Boker, S.; Xie, G.; Maes, H. Mx: Statistical Modeling. 6. Richmond, VA: Department of Psychiatry, Virginia Commonwealth University, Richmond, VA; 2003.

Neale MC, Aggen SH, Maes HH, Kubarych TS, Schmitt JE. Methodological issues in the assessment of substance use phenotypes. Addictive Behaviors 2006a;31:1010-1034. [PubMed: 16723188]

Neale MC, Harvey E, Maes H, Sullivan PF, Kendler KS. Extensions to the modeling of initiation and progression : applications to substance use and abuse. Behavior Genetics 2006b;36:507-524. [PubMed: 16770695]

Neale MC, Lubke G, Aggen SH, Dolan CV. Problems with using sumscores for estimating variance components: contamination and measurement noninvariance. Twin Research and Human Genetics 2005;8:553-568. [PubMed: 16354497]

Pike KM, Wilfley D, Hilbert A, Fairburn CG, Dohm FA, Striegel-Moore R. Antecedent life events of binge-eating disorder. Psychiatry Research 2006;142:19-29. [PubMed: 16713629]

Plomin, R.; DeFries, JC.; McClearn, GE.; McGuffin, P. Behavioral Genetics. New York, NY: Worth Publishers; 2008.

Reichborn-Kjennerud T, Bulik CM, Tambs K, Harris JR. Genetic and environmental influences on binge eating in the absence of compensatory behaviors : a population-based twin study. International Journal of Eating Disorders 2004;36:307-314. [PubMed: 15478129]

Reichborn-Kjennerud T, Bulik CM, Kendler KS, Roysamb E, Maes H, Tambs K, Harris JR. Gender differences in binge-eating: a population-based twin study. Acta Psychiatrica Scandinavica 2003;108:196-202. [PubMed: 12890274]

Striegel-Moore RH, Bulik CM. Risk factors for eating disorders. American Psychologist 2007;62:181198. [PubMed: 17469897]

Striegel-Moore RH, Franko DL. Should binge eating disorder be included in the DSM-V? A critical review of the state of the evidence. Annual Review of Clinical Psychology 2008;4:305-324.

Striegel-Moore RH, Kearney-Cooke A. Exploring parents' attitudes and behaviors about their children's physical appearance. International Journal of Eating Disorders 1994;15:377-85. [PubMed: 8032352]

Sullivan PF, Eaves LJ. Evaluation of analyses of univariate discrete twin data. Behavior Genetics 2002;32:221-227. [PubMed: 12141783]

Van den Berg SM, Glas CAW, Boomsma DI. Variance decomposition using an IRT measurement model. Behavior Genetics 2007;37:604-616. [PubMed: 17534709]

Wade TD, Bergin JL, Martin NG, Gillespie NA, Fairburn CG. A transdiagnostic approach to understanding eating disorders. Journal of Nervous and Mental Disease 2006;194:510-517. [PubMed: 16840847] 


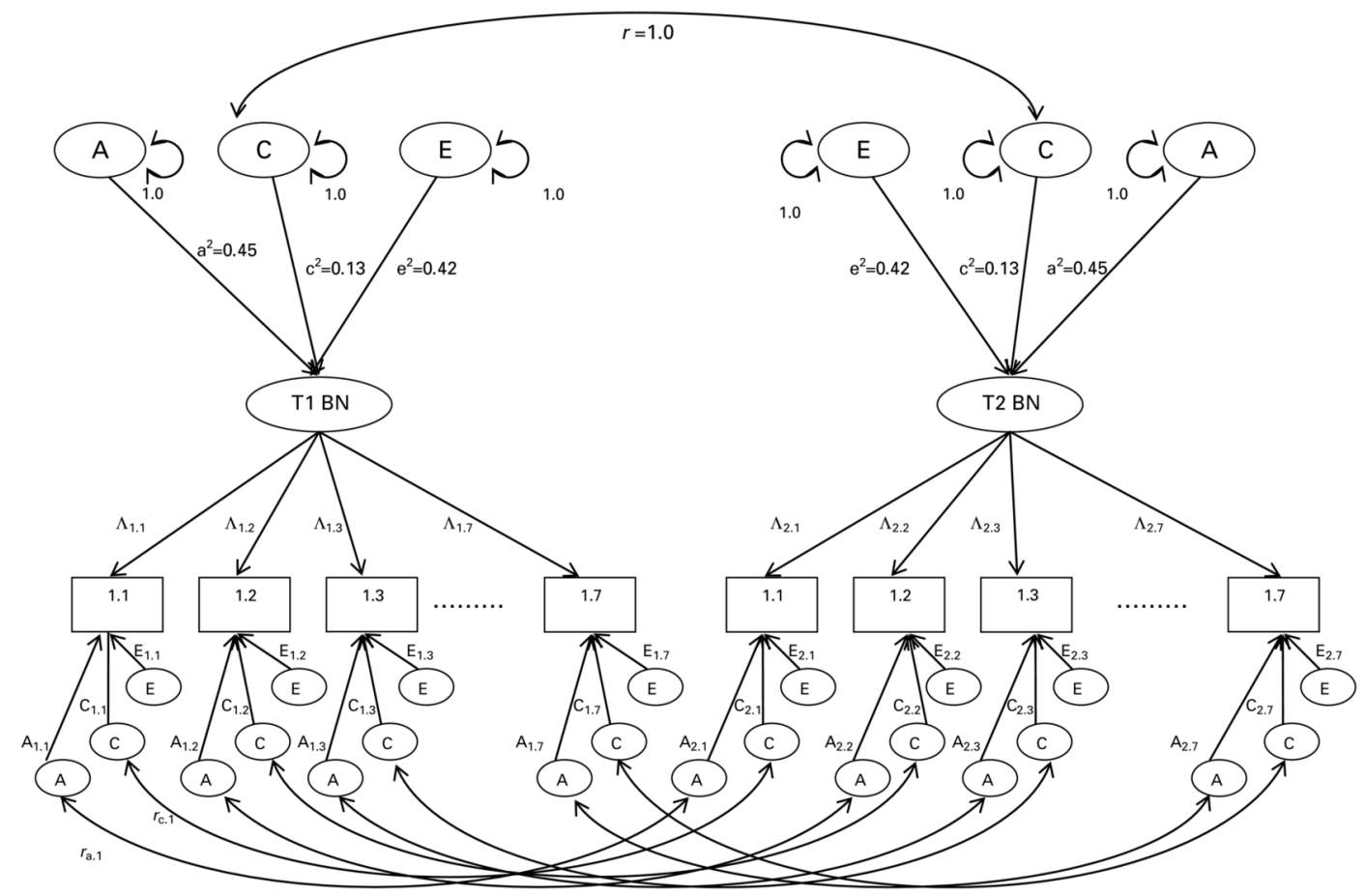

Fig. 1.

Only four items per twin are shown. $\Lambda_{1.1}$ denotes the factor loading for the first item, for twin 1. $\mathrm{A}_{1.1}$ denotes the residual variance due to $\mathrm{A}$ for the first item, for twin $1 . r_{\mathrm{a} .1}=1.0$ or 0.5 and denotes the twin correlation for the residual components due to A for the first item, etc. $r_{\mathrm{c} .1}=1.0$ and denotes the twin correlation for the residual components due to $\mathrm{C}$ for the first item, etc. 
Table 1

BED item numbers, corresponding interview questions, and scoring

\begin{tabular}{|c|c|c|}
\hline Item no. & Interview question & Possible response (frequency) \\
\hline $1^{*}$ & $\begin{array}{l}\text { Have you ever had eating binges when you ate what most people would describe as an unusually } \\
\text { large amount of food in a short time? }\end{array}$ & $\begin{array}{l}\text { (0) No (814) } \\
\text { (1) Yes (207) }\end{array}$ \\
\hline 2 & When you were bingeing the most, how many binges would you have in a month? & $\begin{array}{l}\text { (0) } 0-1 \text { (44) } \\
\text { (1) } 2-7 \text { (99) } \\
\text { (2) } \geq 8 \text { (64) }\end{array}$ \\
\hline 3 & When you were having eating binges, did you feel that your eating was out of control? & $\begin{array}{l}\text { (0) Not at all (33) } \\
\text { (1) Slightly (26) } \\
\text { (2) Somewhat (65) } \\
\text { (3) Very much (61) } \\
\text { (4) Extremely (44) }\end{array}$ \\
\hline 4 & For how long did you have binge-eating episodes? & $\begin{array}{l}\text { (0) Less than one month (62) } \\
\text { (1) } 1 \text { to } 2 \text { months (19) } \\
\text { (2) } 3 \text { months to } 5 \text { months (14) } \\
\text { (3) } 6 \text { months to } 1 \text { year ( } 40) \\
\text { (4) Longer than one year (68) }\end{array}$ \\
\hline 5 & How upset or distressed did binge eating usually make you feel? & $\begin{array}{l}\text { (0) Not at all (20) } \\
\text { (1) Slightly (21) } \\
\text { (2) Moderately (50) } \\
\text { (3) Very much (66) } \\
\text { (4) Extremely (53) }\end{array}$ \\
\hline \multicolumn{3}{|c|}{ Item 6 is the total of the following items, which began with the stem: 'During eating binges, did you ...' } \\
\hline & Eat much more rapidly than usual? & $\begin{array}{l}\text { (0) No (83) } \\
\text { (1) Yes (118) }\end{array}$ \\
\hline & Eat until you felt uncomfortably full? & $\begin{array}{l}\text { (0) No (26) } \\
\text { (1) Yes (179) }\end{array}$ \\
\hline & Eat large amounts of food when you didn't feel physically hungry? & $\begin{array}{l}\text { (0) No (34) } \\
\text { (1) Yes (170) }\end{array}$ \\
\hline & Eat alone because you were embarrassed by how much you were eating? & $\begin{array}{l}\text { (0) No (93) } \\
\text { (1) Yes (109) }\end{array}$ \\
\hline & Feel disgusted with yourself, depressed, or very guilty after overeating? & $\begin{array}{l}\text { (0) No (30) } \\
\text { (1) Yes (172) }\end{array}$ \\
\hline
\end{tabular}

Item 7 is the total of the following items, which began with the stem: 'Have you ever done or used the following during a time when you were binge eating?
Make yourself vomit
(0) No (38)
Laxatives
(1) Yes (171)
Diuretics (water pills)
(0) No (56)
(0) No (40)
Diet pills (over the counter or prescription)
(1) Yes (169)
Exercise more than 2 hours per day
(0) No (94)
(0) No (39)
(1) Yes (169)
Fast or not eat (for 24 hours or more)
(0) No (53)
(1) Yes (155)

* If participants indicated that they had never binged, they skipped to the next section of the questionnaire. 
